\title{
Molecular modelling of dendrimers for nanoscale applications
}

\author{
Tahir Çağın, Guofeng Wang, Ryan Martin, Nicholas Breen and \\ William A Goddard III
}

California Institute of Technology, Materials and Process Simulation Center, (139-74), Division of Chemistry and Chemical Engineering, Pasadena, CA 91125, USA

E-mail: tahir@wag.caltech.edu

Received 2 March 2000

\begin{abstract}
Dendrimers are well defined, highly branched macromolecules that radiate from a central core and are synthesized through a stepwise, repetitive reaction sequence that guarantees complete shells for each generation, leading to polymers that are monodisperse. The synthetic procedures developed for dendrimer preparation permit nearly complete control over the critical molecular design parameters, such as size, shape, surface/interior chemistry, flexibility, and topology. Recent results suggest that dendritic polymers may provide the key to developing a reliable and economical fabrication and manufacturing route to functional nanoscale materials that would have unique properties (electronic, optical, opto-electronic, magnetic, chemical, or biological). In turn, these could be used in designing new nanoscale devices. In this paper, we determine the $3 \mathrm{D}$ molecular structure of various dendrimers with continuous configurational Boltzmann biased direct Monte Carlo method and study their energetic and structural properties using molecular dynamics after annealing these molecular representations.
\end{abstract}

(Some figures in this article are in colour only in the electronic version; see www.iop.org)

\section{Introduction}

Dendrimers and hyperbranched polymers represent a novel class of structurally controlled macromolecules derived from a branches-upon-branches structural motif [1,2]. Dendrimers are well defined, highly branched macromolecules that radiate from a central core and are synthesized through a stepwise, repetitive reaction sequence that guarantees complete shells for each generation, leading to polymers that are monodisperse [3]. The synthetic procedures developed for dendrimer preparation permit nearly complete control over the critical molecular design parameters, such as size, shape, surface/interior chemistry, flexibility, and topology [1-3]. Synthetic techniques that have proved effective include the Starburst divergent strategy of Tomalia and co-workers $[1,2]$, the convergent growth strategy of Fréchet and co-workers [4-6], and the self-assembly strategy of Zimmerman and co-workers [7]. These methods have proved effective in generating macromolecules with a unique combination of properties [8-11].

The geometric characterization of dendrimer structure has lagged behind this rapid progress in synthesis and design. The problem is that these molecules possess an enormous number of energetically permissible conformations, and in solution there is rapid interchange between them. The diffraction techniques yield little structural information. In addition, a number of generations involve the same monomers, making it difficult to extract precise information about the local structure from infrared or NMR experiments. Thus the most precise experimental data about overall structure comes from size exclusion chromatography. The main experimental data about the geometric character of particular sites has come from NMR relaxation times for molecules able to partially penetrate the dendrimer [12].

A particular advantage of using theory is that the properties of new materials can be predicted in advance of experiments. This allows the system to be adjusted and refined (designed) so as to obtain the optimal properties before the arduous experimental task of synthesis and characterization. However, there are significant challenges in using theory to predict accurate properties for functional dendritic materials.

Below we describe some recent developments in the area of dendrimers and molecular modelling applications to a list of dendritic polymers: PAMAM, stimuli responsive polymers, colloidal crystals of self-assembled dendrimers and cored dendritic architectures for molecular imprinting. The paper is organized as follows. In section 2, we will briefly describe the continuous configurational Boltzmann biased (CCBB) direct Monte Carlo (MC) method [13, 14] which is applied in building $3 \mathrm{D}$ molecular representations of the dendrimers used in this study. In section 3 we describe the molecular dynamics simulation on various dendrimers. 


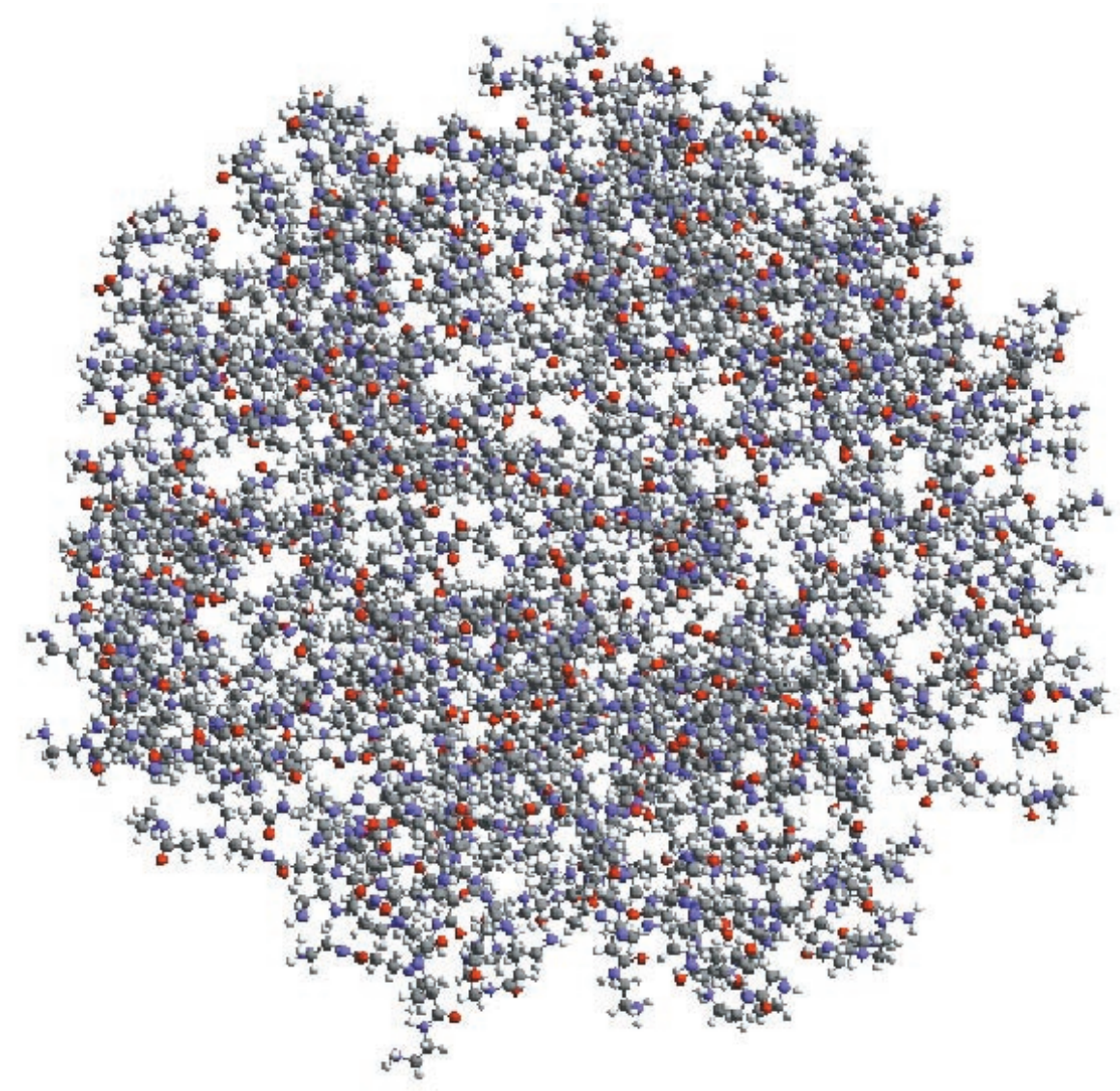

Figure 1. CPK view of the 3D annealed structure of the EDA-cored generation 7 PAMAM dendrimer.

\section{The ССВB direct $M C$ method for dendrimers}

To predict the properties of polymers, it is necessary to determine an ensemble of conformations highly populated at the temperature and pressure of interest. An efficient method for predicting these conformations uses MC sampling. The CCBB MC is an improved method which was developed to serve this purpose $[13,14]$. Here, we have taken advantage of this method to generate energetically preferrable 3D molecular structures of various dendrimers.

The CCBB direct MC method is developed on the basis of the independent rotational sampling (IRS) method. In the IRS method, torsional degrees of the polymer chains are sampled using a weighting function based on the Boltzmann factor of the torsion energy. The normalized torsion weighting function (TWF), $W_{\text {IRS }}$, for IRS is defined as

$$
W_{\mathrm{IRS}}=\frac{g_{\mathrm{IRS}}(\phi)}{z_{\mathrm{IRS}}}
$$

where,

$$
\begin{gathered}
z_{\mathrm{IRS}}=\int_{0}^{2 \pi} g_{\mathrm{IRS}}(\phi) \mathrm{d} \phi \\
g_{\mathrm{IRS}}(\phi)=\exp \left[-\beta E_{t}(\phi)\right] .
\end{gathered}
$$

However, the possibility of spatial overlaps between nonbonded atoms cannot be excluded in IRS. Thus, configurations with extremely high energy will be generated.
In order to remedy this, nonbonding interactions in the vicinity of the growing chain end and within a cutoff sphere are included in the TWF calculation. Hence, the Boltzmann factor for the nonbond energy within a cutoff radius, $R_{\mathrm{C}}$, is also accounted for in the definition of TWF, $W_{\mathrm{CCB}}$, as

$$
W_{\mathrm{CCB}}\left(\phi_{i} ; \phi_{1}, \ldots, \phi_{i-1}\right)=\frac{g_{\mathrm{CCB}}\left(\phi_{i} ; \phi_{1}, \ldots, \phi_{i-1}\right)}{z_{\mathrm{CCB}}\left(\phi_{1}, \ldots, \phi_{i-1}\right)}
$$

where,

$$
\begin{aligned}
& z_{\mathrm{CCB}}\left(\phi_{1}, \ldots, \phi_{i-1}\right)=\int_{0}^{2 \pi} g_{\mathrm{CCB}}\left(\phi_{i} ; \phi_{1}, \ldots, \phi_{i-1}\right) \mathrm{d} \phi_{i} \\
& g_{\mathrm{CCB}}\left(\phi_{i} ; \phi_{1}, \ldots, \phi_{i-1}\right) \\
& =g_{\mathrm{IRS}}\left(\phi_{i}\right) \exp \left[-\beta \sum_{j, k} \Theta\left(R_{\mathrm{C}}-r_{j k}\right) E_{L J}\left(r_{j k}\right)\right]
\end{aligned}
$$

where atom $j$ belongs to the growing chain end group and atom $k$ is one of the atoms in the polymer chain grown. $\Theta(R)$ is the Heavyside step function, $\Theta(R)=0$ when $R<0$ and $\Theta(R)=1$ when $R \geqslant 0$.

Prior to each step of chain sampling, the torsion energy and nonbond energy within the cutoff radius of the growing end were calculated for a fixed number of grid points (equally spaced from 0 to $2 \pi$ ) and $W_{\mathrm{CCB}}$ was evaluated. The auxiliary distribution, $P_{\mathrm{CCB}}(\phi)$, is defined as

$$
P_{\mathrm{CCB}}(\phi)=\int_{0}^{\phi} W_{\mathrm{CCB}}\left(\phi^{\prime}\right) \mathrm{d} \phi^{\prime} .
$$


Table 1. Variation of radius of gyration at $300 \mathrm{~K}$ as a function of generation and with amine and EDA.

\begin{tabular}{lrr}
\hline Generation & \multicolumn{1}{l}{$R_{\mathrm{g}}(\AA)$} & \multicolumn{1}{l}{$R_{\mathrm{g}}(\AA)$} \\
\hline 1 & 4.971490 & 3.774253 \\
2 & 7.034312 & 6.030406 \\
3 & 9.774769 & 8.409326 \\
4 & 13.016935 & 11.155713 \\
5 & 16.355944 & 16.001749 \\
6 & 21.668465 & 20.598940 \\
7 & 27.624895 & 26.454330 \\
\hline
\end{tabular}

A random number $\xi$, uniformly distributed in the interval $[0,1)$, is drawn and the torsion angle $\phi_{i}$ is obtained by requiring

$$
P_{\mathrm{CCB}}\left(\phi_{i}\right)=\int_{0}^{\phi_{i}} W_{\mathrm{CCB}}(\phi) \mathrm{d} \phi=\xi .
$$

\section{Results}

\subsection{PAMAM: structure as a function of generation}

Recently, dendritic polymers have been used as soluble templates/unimolecular reactors from which nanoclusters of inorganic compounds or elements can be synthesized. The basic concept involves using dendrimers as hosts to preorganize small molecules or metal ions, followed by a simple in situ reaction which will immobilize and stabilize domains of atomic or molecular guest components (inorganic compounds as well as elemental metals) [15]. In one of these examples poly(amidoamine) (PAMAM) dendrimers have been used, to attract copper (II) ions inside the macromolecules where they are subsequently reacted with solubilized $\mathrm{H}_{2} \mathrm{~S}$ to form metal sulfides [16]. These organic/inorganic, dendrimer-based hybrid species have been termed 'nanocomposites' and display unusual properties. For example, the solubility of the nanocomposites is determined by the properties of the host dendrimer molecules. This allows for solubilization of the inorganic guest compounds in environments in which they are inherently insoluble. Since it has been established that there is no covalent bond between host and guest, these observations suggest that the inorganics are physically and spatially restricted by the dendrimer shell. However, this structure has not been verified. Here we use 3D structure building tools based on the CCBB MC method and molecular dynamics techniques to investigate the structural characteristics of PAMAM dendrimers.

We carried out molecular dynamics simulations at room temperature to investigate the structure of the PAMAM dendrimers upto generation 7. We have used two different initiators, ethylenediamine (EDA) and ammonia $\left(\mathrm{NH}_{3}\right)$. Figure 1 shows the structure of the PAMAM-EDA generation 7 dendrimer.

In the molecular model building process, we used an annealing scheme which uses successive steps of energy minimization and molecular dynamics runs. After constructing initial structures, molecular dynamics simulations are carried out at $T=300 \mathrm{~K}$ using Dreiding force fields [17]. Each generation (including the half-generation simulations) was carried out over 200 ps (with a time step of $1 \mathrm{fs}$ ). The initial
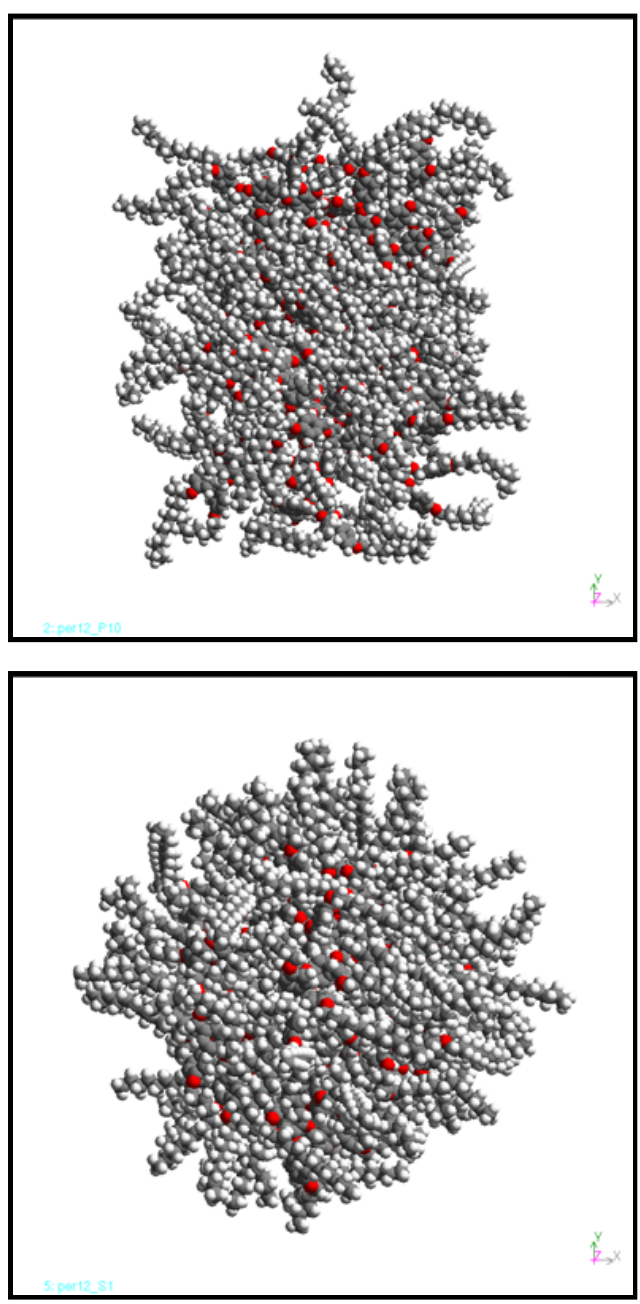

Figure 2. Dendritic supramolecular assemblies built using the CCCB method: (a) 2-functional monomer unit resulting in cylindirical assembly, upon crystallization this results in a two-dimensional crystal structure in space group p6mm; (b) 3-functional monomer unit resulting in spherical assembly, upon crystallization this supramolecular assembly leads to an A-15 crystal structure.

$100 \mathrm{ps}$ of the run is treated as equilibration. The structural analysis was carried over the last 100 ps of each simulation. In table 1 we list the calculated radius of gyration for generations $1-7$.

\subsection{Supramolecular assemblies of dendrimers: colloidal crystals and discotic liquid crystals}

Recently, Percec and co-workers reported the synthesis of three generations of self-assembling monodendrons based on die $\mathrm{AB}(3)$ building block methyl 3,4,5trihydroxybenzoate [18]. The first 3,4,5-tris[p- $(n-$ dodecan-1-yloxy)benzyloxy]benzoic acid and the secondgeneration methyl 3,4,5-tris $\left\{3^{\prime}, 4^{\prime}, 5^{\prime}\right.$-tris[p-(n-dodecan-1yloxy)benzyloxy]benzyloxy $\}$ benzoate monodendrons selfassemble into cylindrical supramolecular dendrimers that self-organize in a two-dimensional p6mm lattice. The thirdgeneration monodendron 3,4,5-tris $\left(3^{\prime}, 4^{\prime}, 5^{\prime}\right.$-tris $\left\{3^{\prime \prime}, 4^{\prime \prime}, 5^{\prime \prime}\right.$ tris[p-(n-dodecan-1-yloxy)benzyloxy] benzyloxy $\}$ benzyloxy)-benzoate self-assembles in a spherical dendrimer that 


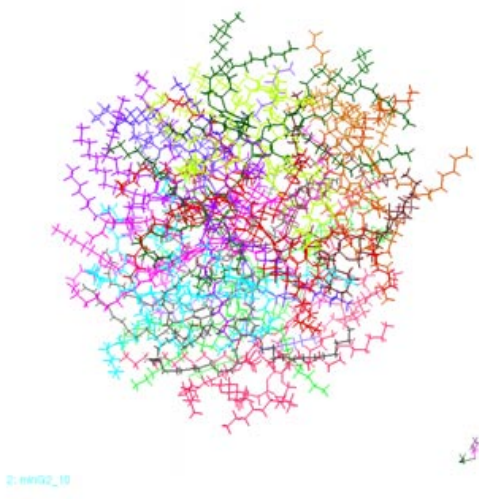

(a)

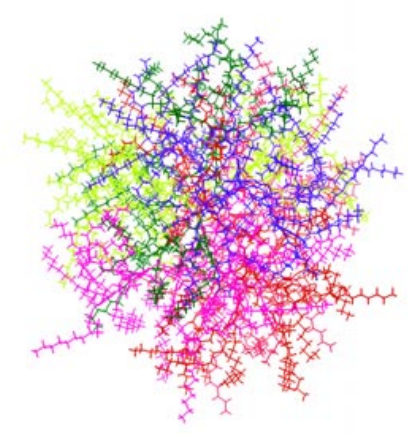

(b)

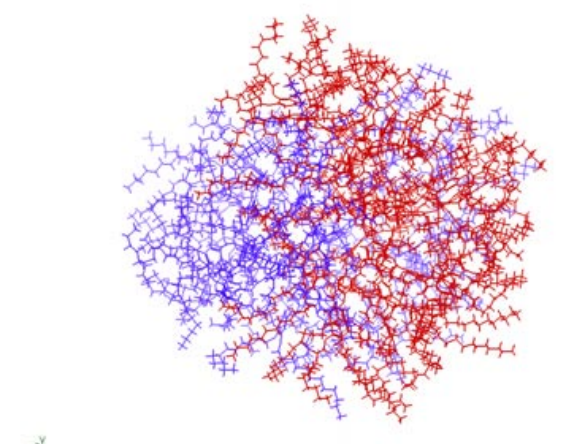

$(c)$

Figure 3. Three generations of Percec dendrimers all leading to spherical supramolecules which crystallize in an A-15 cubic structure: (a) generation 2, (b) generation 3 and (c) generation 4.

Table 2. Theoretical number of monodendrons, molecular weight, number of atoms and number of end groups for the $(3,4,5) n 12 \mathrm{G} n$ - $\mathrm{COOH}$ spherical supramolecule: $\mu$ is the number of monodendrons, $M_{\mathrm{w}}$ the molecular weight of the spherical supramolecular dendrimer, $N_{\mathrm{A}}$ the number of atoms, $N_{\mathrm{C}}, N_{\mathrm{H}}, N_{\mathrm{O}}$, the number of $\mathrm{C}, \mathrm{H}$ and $\mathrm{O}$ atoms respectively and $N_{\mathrm{E}}$ the number of the end groups in the supramolecule.

\begin{tabular}{llllllll}
\hline Generation & $\mu$ & $M_{\mathrm{w}}$ & $N_{\mathrm{A}}$ & $N_{\mathrm{C}}$ & $N_{\mathrm{H}}$ & $N_{\mathrm{O}}$ & $N_{\mathrm{E}}$ \\
\hline 2 & 12 & 25192.6 & 4680 & 1632 & 2880 & 168 & 108 \\
3 & 6 & 38234.1 & 7092 & 2490 & 4356 & 246 & 162 \\
4 & 2 & 38382.3 & 7116 & 2504 & 4368 & 244 & 162 \\
\hline
\end{tabular}

Table 3. Average radius of gyration, $R_{\mathrm{g}}$, average volumetric radii, $R_{\mathrm{v}}$, which are calculated from the molecular volume using $V=4 / 3 \pi R_{\mathrm{v}}^{3}$ and the average lattice parameters of the $(3,4,5) n 12 \mathrm{G} n-\mathrm{COOH}$ spherical supramolecule.

\begin{tabular}{llllllllll}
\hline & \multicolumn{2}{c}{$R_{\mathrm{g}}(\AA)$} & & \multicolumn{2}{c}{$R_{\mathrm{v}}(\AA)$} & & \multicolumn{3}{c}{$a=4 R_{\mathrm{v}}(\AA)$} \\
\cline { 2 - 3 } \cline { 8 - 10 } Generation & CCBB & Theory & & CCBB & Theory & & CCBB & Theory & Expt. \\
\hline 2 & 13.84 & 17.19 & & 16.91 & 18.77 & & 67.66 & 75.07 & 68.3 \\
3 & 16.84 & 19.82 & & 19.80 & 21.56 & & 79.21 & 86.22 & 79.2 \\
4 & 18.28 & 20.55 & & 20.32 & 21.59 & & 80.91 & 86.35 & 84.0 \\
\hline
\end{tabular}

self-organizes in a $3 \mathrm{D}$ cubic $\operatorname{Pm} \overline{3} n$ lattice. Structural analysis of these lattices by $\mathrm{x}$-ray diffraction provided the first direct demonstration of the supramolecular dendrimer shape change from cylindrical to spherical and indirect determination of the average shape change of the monodendron from a quarter of a disc to a half of a disc and to a sixth of a sphere as a function of generation number. These results have demonstrated the concept of monodendron and supramolecular dendrimer shape control by generation number.

As depicted in figure 2, dendrimers can be designed to aggregate to form cylinders, $(a)$, or spheres, $(b)$, depending upon the nature of the fundamental building unit. One class of these systems leads to cone-type dendrimers that organize into spheres ( 2 to 12 per sphere depending on generation) which then pack into the unusual $\operatorname{Pm} \overline{3} n$ (A-15) type cubic crystal structure having spheres at the corners and body centre of the cube, plus two on each face.

Using the CCBB technique, we have built structures for generation 2-4 dendrimers. We then carried out successive minimization and dynamics simulations to anneal the structures to obtain stable structures. Figure 3(a) displays the generation 2 dendrimer, figure $3(b)$ shows generation 3 and, finally, figure $3(c)$ shows the generation 4 . Tables 2 and 3 list the physical properties of these dendrimers. We have also determined the variation of density as a function of distance from the centre of mass of the dendrimer, see figure 4. We then carried out large-scale MD calculations on this system to detemine the equilibrium structure at room temperature. These are large systems with 37000 atoms (excluding hydrogen) per unit cell of $\mathrm{Pm} \overline{3} n$ structure. The equilibrated structures led to $\mathrm{x}$-ray diffraction intensities in good agreement with experiment, see figure 5.

The self-assembly of this system represents a model problem for a coarsening approach starting from classical atomic level simulation and averaging over atoms to obtain a supramolecular representation. To do this, we performed molecular dynamics simulation on an isolated single spherical assembly at $300 \mathrm{~K}$. We also performed successive simulations with two spherical supramolecular assemblies as the distance between the sphere centres varied until they overlapped. From these simulations we determined the interaction energetics of a pair of spherical assemblies. The interaction energy can be approximated by a flat-bottom Morse potential. The inner core and dispersion parts have the same depth, but different minima. Preliminary studies using this force field gave rise to stable $\operatorname{Pm} \overline{3} n$ structure [19]. 


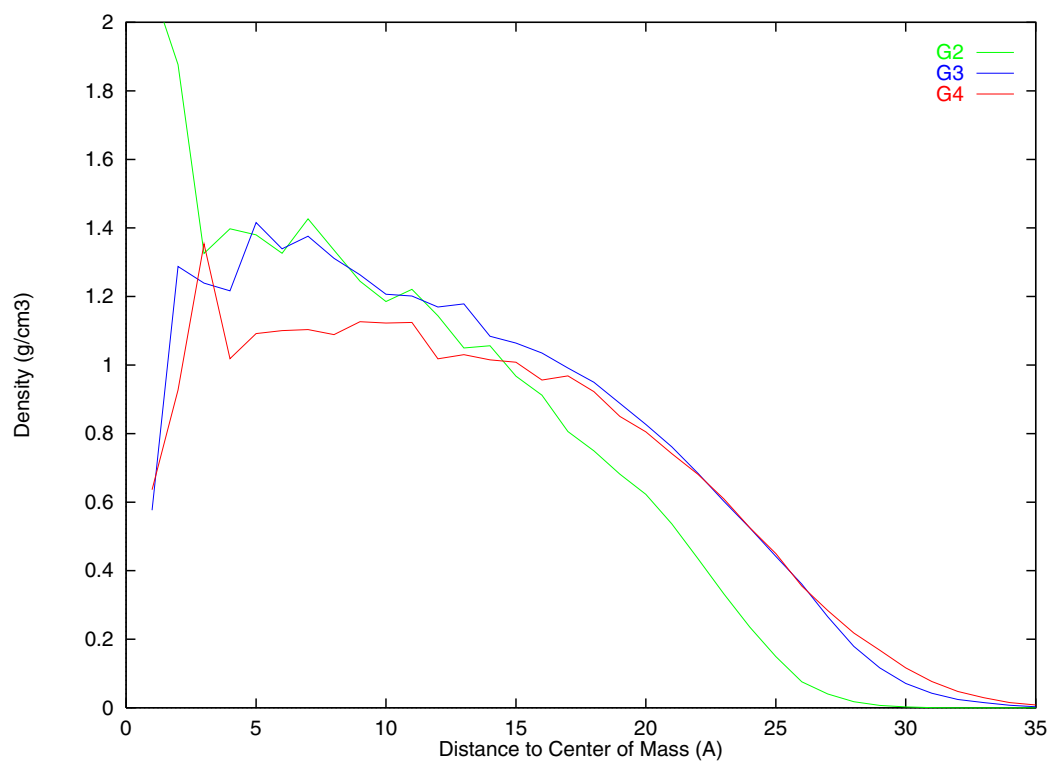

Figure 4. Variation of density as a function of distance from the centre of mass of the spherical dendritic assemblies for generations $2-4$.

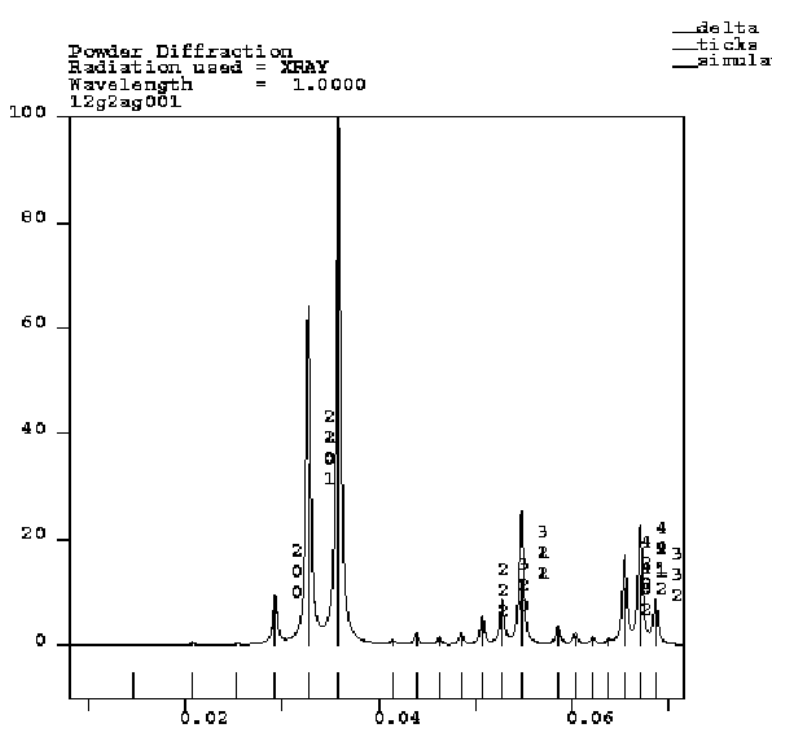

Figure 5. Theoretical x-ray diffraction pattern calculated from the crystal structure containing eight spherical supramolecular assemblies per unit cell.

\subsection{Stimuli-responsive hybrid macromolecules}

Frechét [5] has prepared new stimuli-responsive macromolecules based on linear, star and dendritic blocks. A novel amphiphilic hybrid macromolecule has been synthesized by Fréchet [6]. This macromolecule has hydrophobic dendritic groups at the periphery of a hydrophilic polyethylene glycol (PEG) star. Light-scattering experiments suggest that changing the solvent from THF (tetrahydrofuran) to methanol leads to large changes in structure. To study the response of these macromolecules to such variations in environment, we used molecular dynamics (MD) to predict structures and properties for the macromolecule in methanol and THF. These calculations used explicit solvent and periodic boundary conditions. The Frechet macromolecule has 2761 atoms and we also in- cluded 26892 methanol molecules or 13140 THF molecules. We used the MPSim MD program [20] to carry out 700 ps of NVT dynamic simulations at $300 \mathrm{~K}$. These results show that in THF the Fréchet hybrid macromolecule has a somewhat compact PEG core with the dendrimer extending outward into the solvent while in methanol the PEG tends to wrap around the dendrimer to shield it from the solvent. These results validate the interpretations by Fréchet [6].

We have first carried out large-scale MD calculations on this system in two different solvents (THF and methanol) to determine the equilibrium structure as a function of solvent and temperature. The structures of the slightly extended and coiled forms of dendrimers are shown in figure 6. The variation of radius of gyration versus time is given in figure 7 .

\subsection{Cored dendrimers for unimolecular imprinting}

The development of unimolecular imprinting is critical in many nanoscale applications ranging from the development of new chemical sensors, electronic, chemical sensing, nanoand molecular-electronics and shape and functional group selective binding, especially for nanoscale catalytic reactors.

Dendrimers, due to their very well defined structures, are the most likely candidates for this purpose. Recently, Zimmerman has proposed dendrimers with homoallyl groups on their periphery [21] which could be linked through a ringclosing methathesis reaction. These dendrimers contain three cleavable ester bonds at their core with robust ether linkages throughout the remaining structure, see figure 8.

The determination and study of the 3D molecular structure of these supramolecular assemblies is a challenging problem. Using the CCBB method we have developed an ensemble of 50 candidate 3D molecular structures, figure 9 displays one member of the ensemble. After annealing these structures using molecular mechanics and molecular dynamics methods we have obtained the final 42 of the 50 cored dendrimer structures with reasonable energies. These structures are then analysed using their final energy as criteria. 
T Çă̆ın et al

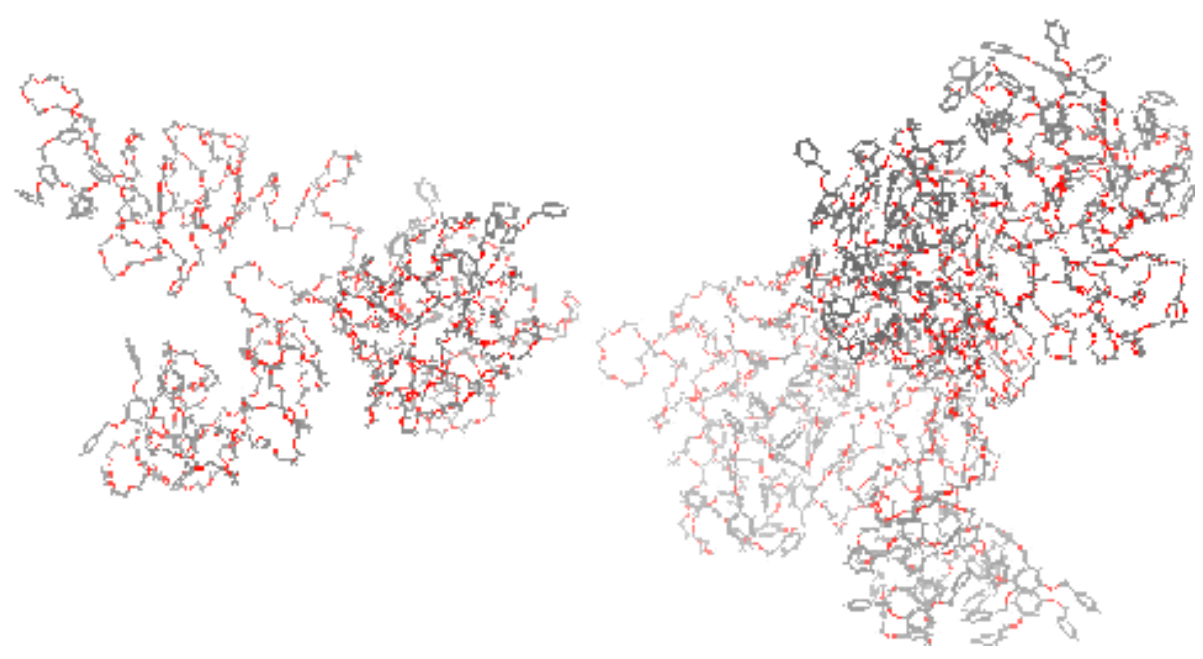

Figure 6. Final structures obtained from MD simulations of the Fréchet hybrid star dendritic polymer in explicit solvent: 84 monomer coil structure (left) and 113 monomer coil structure (right).

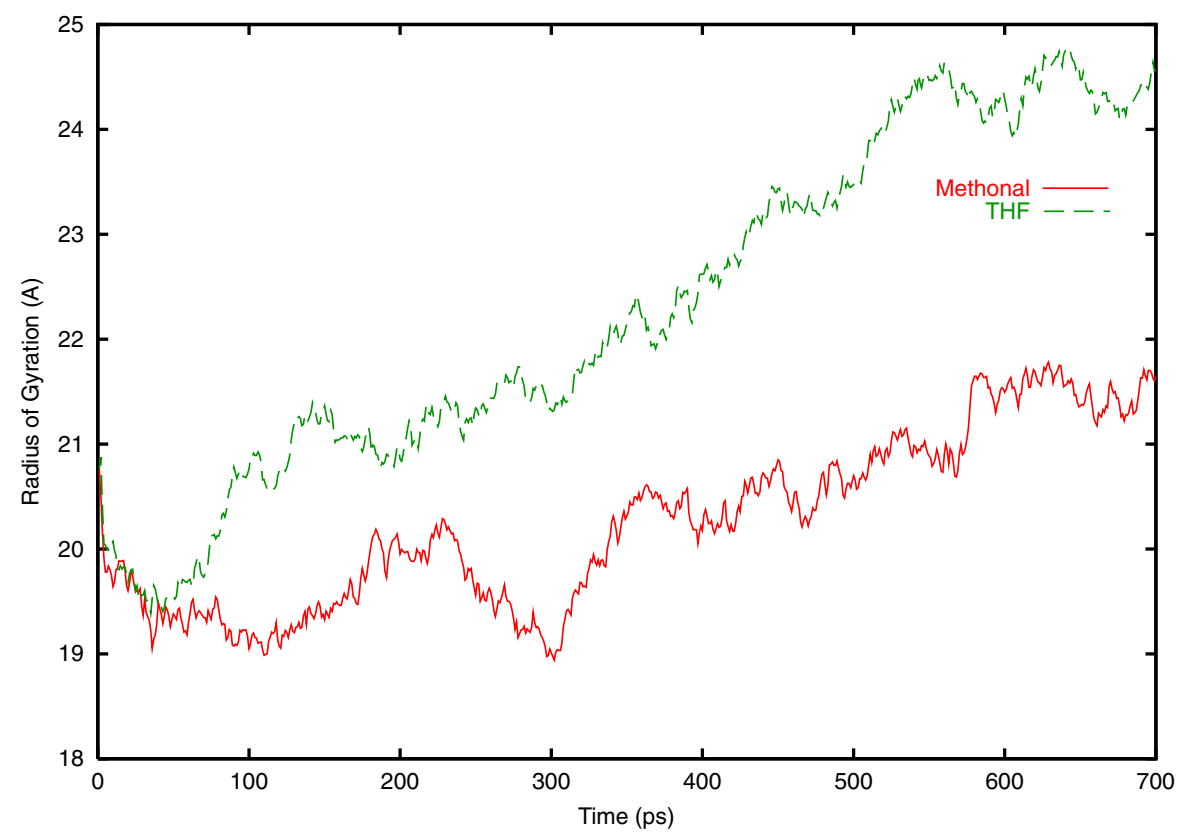

Figure 7. The variation of radius of gyration as a function of time for the star-dendritic hybrid stimuli responsive macromolecule in two different solvents (methanol and THF).

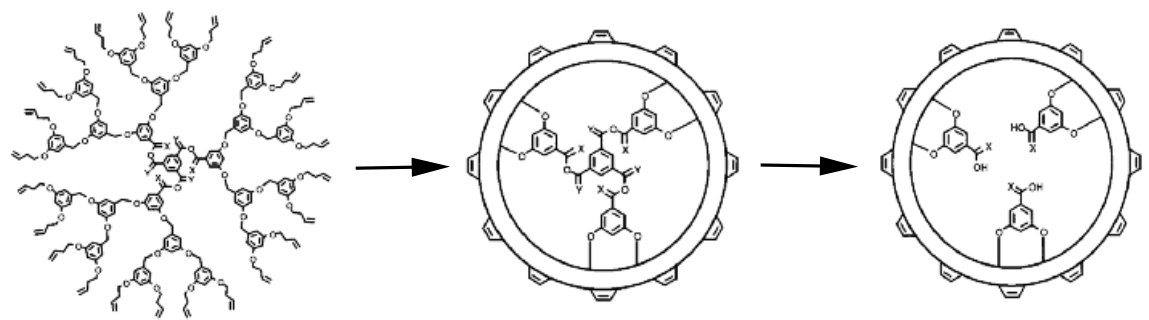

Figure 8. Schematic representation of the ring-closing metathesis reaction for the cored dendrimers of Zimmerman and co-workers.

The energy distribution for the final 42 structures is given in figure 10 . The average radius of gyration for the ensemble of 42 molecules is $15.93 \AA$. The standard deviation of the radius of gyration distribution is only $0.42 \AA$.

We have used these 42 structures to study the ring closing through a crosslinking algorithm. In this algorithm each structure was annealed (2 ps dynamics/40 steps minimization), and crosslinked (at most one pair per round, within $5 \AA$ A of each other), for 25 cycles, then minimized for up to 1000 steps. Each sample molecule was run twice, creating 


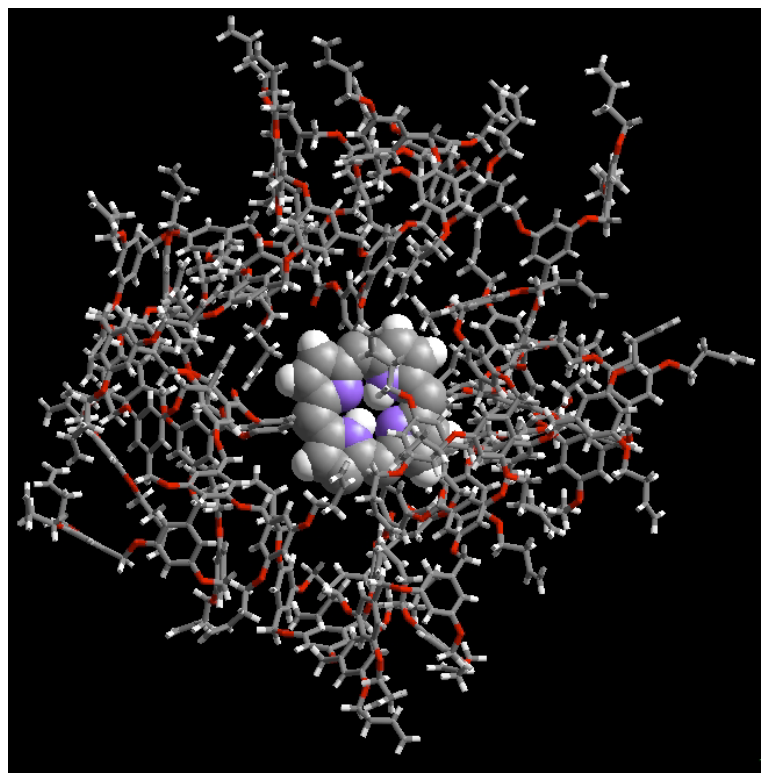

Figure 9. A 3D model structure of the cored dendrimer after the CCBB building procedure.

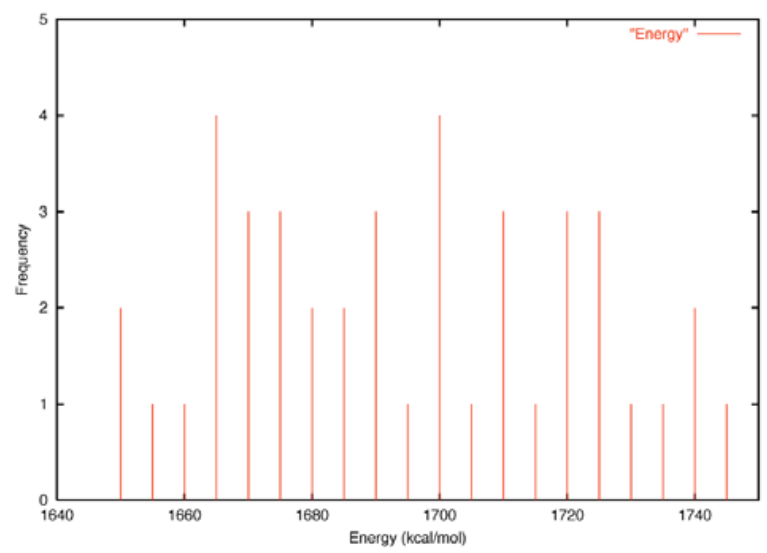

Figure 10. The energy distribution analysis of the cored-dendrimer structures obtained from the CCBB direct MC method building procedure. The analysis is performed over 42 molecules.

a total of 84 data sets. The second modification does appear to be more thermodynamically realistic, as the bonds that it disallows are of high energy - the first set showed increasing total energy with increasing bonding, while the second set instead decreases as it is expected.

Without the modification, $55.8 \%$ of bonding took place between wedges (one of the four segments extending from the core) and $44.2 \%$ took place within a wedge. With the modification, these percentages become $52.9 \%$ and $47.1 \%$. There appears to be no substantial energy difference between any particular set of bonds (with the exception of the pairs disallowed by the algorithm).

The frequency distribution peaks at 12 bond pairs formed, see figure 11. The experimental results via mass spectroscopy peaked at about 11 or 12 . The distribution we have generated is broader than the mass spectroscopy experiments, and appears to favour some higher levels of bonding.
Frequency distribution - crosslinking of Zimmerman dendrimers

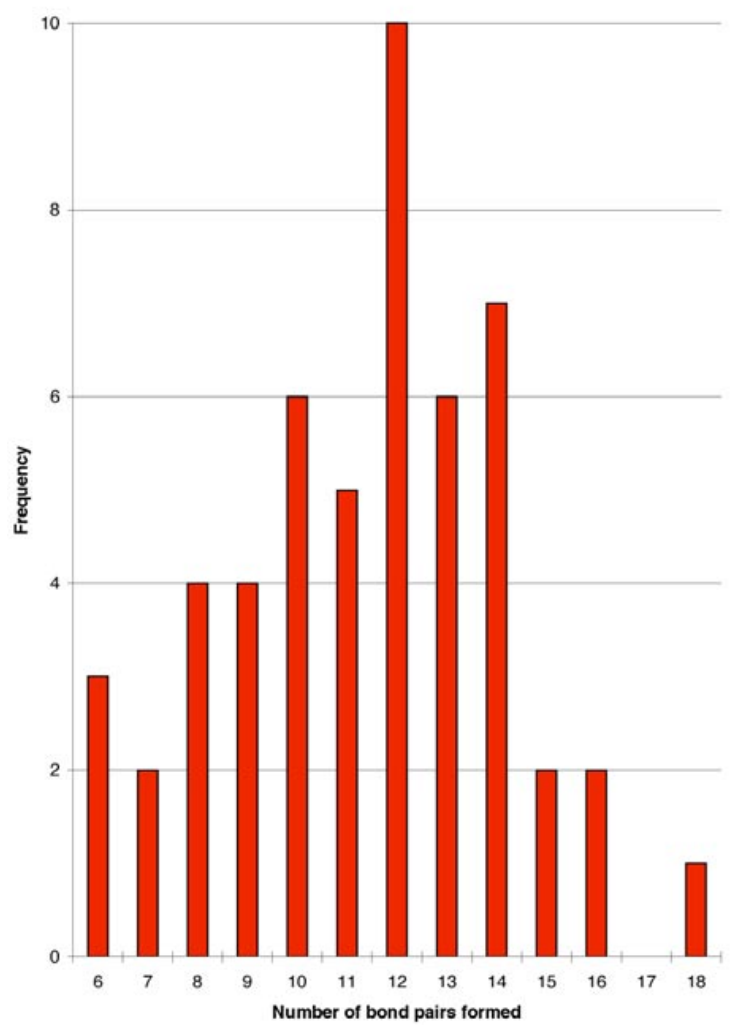

Figure 11. The distribution of bond formation for cored dendrimers.

All structures appear to be intact even when the core is removed, meaning they have enough cross-wedge linking to hold together. This is in good agreement with experimental results, which report a nearly quantitative yield after coring.

\section{Discussions}

To conclude, we would like to address some of the connections between dendrimers and technological applications, especially at the nanotechnology level.

Dendrimers are a new class of 3D, man-made molecules produced by an unusual synthetic route which incorporates repetitive branching sequences to create a unique novel architecture. Exceptional features of the dendritic architecture include a high degree of structural symmetry, a density gradient displaying an intra-molecular minimum value and a well defined number of terminal groups which may be chemically different from the interior [22]. The combination of these features creates an environment within the dendrimer molecule which facilitates the developement of reliable and economical functional nanoscale materials which would have unique properties (electronic, optical, opto-electronic, magnetic, chemical, or biological) which could be the basis for new nanoscale technology and devices.

Dendrimer technology has been established to obtain cone-shaped, spherical, or disc-like shapes that are monodisperse with sizes in the range 2-12 nm [9-11, 15]. These structures can be designed to be containers for organic 
dye molecules [23] or for metal or semiconductor clusters, with exteriors that dissolve them in appropriate media or stick them onto appropriate surfaces. The sequestered metal clusters of $\mathrm{Fe}$ and $\mathrm{Co}$ show magnetic properties and the sequestered semiconductor probably behave as spherical quantum dots.

The metals could also be prepared pure and the surfaces oxidized to give protection or to provide a barrier for electronic purposes. The cones, spheres, or discs can be covalently attached at regular spaces to polymer backbones to form linear necklaces. Thus one can imagine a regular array of metals or quantum dots whose spacing and diameter can be controlled exactly. These linear necklaces can be directed to associate in bundles which could then be cross-linked to provide stable 2D networks of metals or quantum dots. Used as a mask this could be the basis for nanolithography with lines of 2 to $5 \mathrm{~nm}$. Used as a detector it could provide a nano channel plate of magnificent resolution. Used as an active electronic element it could provide for entirely new types of devices.

The current magnetic clusters are magnetically soft, which could be useful for some applications. Placing a cap on the dendrimer that interacts strongly with the metal surface could increase their coercive force. Using appropriate dendrimers the shape of the metal could be changed to disc-like or cylindrical, providing the possibility of use as a magnetic storage medium. The dendrimers can be designed so that the interior or exterior is hydrophobic or hydrophilic and rigid or flexible and possessing small or large pores. This provides enormous opportunities for chemical sensors.

\section{Acknowledgments}

The research projects reported in this paper are supported by grants ARO-MURI, ARO-AASERT and ARO-DURIP. The facilities of MSC is also supported by funds from NSF (CHE 95-22179), DOE-ASCI, NASA/Ames, Avery Dennison, BP Chemical, Beckman Institute, Chevron Petroleum Technology Co, Chevron Chemical Co, Dow Chemical, Exxon, and Seiko-Epson.

\section{References}

[1] Tomalia D A, Naylor A M and Goddard W A III 1990 Starburst dendrimers-molecular level control of size, shape surface chemistry, topology and flexibility from atoms to macroscopic matter Angew. Chem. Intl. Edit 29 $138-75$

[2] Naylor A M, Goddard W A III, Keiffer G E and Tomalia D A 1989 Starburst dendrimers 5 molecular shape control $J$. Am. Chem. Soc. $1112339-41$

[3] Tomalia D A, Hedstrand D M and Ferritto M S 1999 Combburst dendrimer topology - new macromolecular architecture derived from dendritic grafting Macromol. 24 $1435-8$

[4] Hawker C J, Wooley K L and Fréchet J M J 1993 Unimolecular micelles and globular amphiphiles-dendritic macromolecules as novel recyclable solubilization agents $J$. Chem. Soc. Perkins Trans. 12 1287-97
[5] Fréchet J M J 1994 Functional polymers and dendrimers-reactivity, molecular architecture and interfacial energy Science $\mathbf{2 6 3} 1710-5$

[6] Fréchet J M J 1995 Self condensing vinyl polymerization - an approach to dendritic materials Science 269 1080-3

[7] Zimmerman S C, Zeng F W, Richert D E C and Kolotuchin S V 1996 Self assembling dendrimers Science 271 1095-8

[8] Bell T W 1996 Molecular trees, a new branch of chemistry Science 271 1077-8

[9] van Hest J C M, Delnoye D A P, Baars M W P L, van Genderen M H P and Meijer E W 1995 Polystyrene-dendrimer amphiphilic block copolymers with generation dependent aggregation Science $\mathbf{2 6 8}$ $1592-5$

[10] Jansen F G A J, Meijer E W and de Brabander-van den Berg E M M 1995 The dendritic box-shape selective liberation of encapsulated guests $J$. Am. Chem. Soc. 117 4417-8

[11] Jansen F G A J, de Brabander-van den Berg E M M and Meijer E W 1995 Encapsulation of guest molecules into a dendritic box Science 266 1226-9

[12] Gopidas K R, Leheny A R, Caminati G, Turro N J and Tomalia D A 1991 Photophysical investigation of similarities between starburst dendrimers and anionic micelles J. Am. Chem. Soc. 113 7335-42

[13] Sadanobu J and Goddard W A III 1997 The continuous configurational Boltzmann biased Monte Carlo method for free energy properties of polymer chains $J$. Chem. Phys. 106 6722-9

[14] Sadanobu J and Goddard W A III 1998 Efficient Monte Carlo method for free energy evaluation of polymer chains Fluid Phase Equil. 144 415-25

[15] Balogh L, Swanson D R, Spindler R and Tomalia D A 1997 Formatiom and characterization of dendrimer based wator soluble inorganic nanocomposites Proc. Am. Chemical Society, Division of Polymeric Materials Science and Engineering (Los Vegas, Nevada, Sept, 1997) vol 77

[16] Balogh L, Swanson D R, Fry J and Tomalia D A 1997 Component transfer without resistance: interactions of copper(II) ions with poly(amidoamine) dendrimers Abst. Pap. Am. Chem. Soc. vol 1216 016-IEC

[17] Mayo S L, Olafson B D and Goddard W A III 1990 Dreiding-A generic force field for molecular simulations J. Phys. Chem. 94 8897-909

[18] Hudson S D, Jung H T, Percec V, Cho W D, Johansson G, Ungar G and Balagurusamy V S K 1997 Direct visualization of individual cylinderical and spherical supramolecular dendrimers Science 278 449-52

[19] Çă̆ın T, Miklis P J, Wang G, Zamanakos G, Martin R, Li H, Mainz D T, Vaidehi N and Goddard W A III 1999 Recent advances in simulation of dendritic polymers Dynamics in Small Confining Systems vol 5 pp 299-310

[20] Lim K T, Brunett S, Iotov M, McClurg R C, Vaidehi N, Dasgupta S, Taylor S and Goddard W A III 1997 Molecular dynamics for very large systems on massively parallel computers: the MPSim program J. Comput. Chem. 18 501-12

[21] Wendland M S and Zimmerman S C 1999 Synthesis of cored dendrimers J. Am. Chem. Soc. 121 1389-90

[22] Lothian-Tomalia M K, Hedstrand D M, Tomalia D A, Padias A B and Hall H K Jr 1997 A contemporary survey of covalent connectivity and complexity. The divergent synthesis of poly(thioether) dendrimers. Amplified, genealogically directed synthesis leading to the de Gennes dense packed state Tetrahedron 53 15 495-513

[23] Miklis P J, Çağın T and Goddard W A III 1997 Dynamics of bengal rose encapsulated in the Meijer dendrimer box $J$. Am. Chem. Soc. 119 7458-62 